LBL-37243

UC-414

Presented at the

7th European Symposium on Semiconductor Detectors

Schloss Elmau, Germany, May 7-10, 1995

\title{
P-TYPE SILICON DRIFT DETECTORS
}

J.T. Walton, B. Krieger, D. Krofcheck, R. O'Donnel,

G. Odyniec, M. Partlan, and N.W. Wang

Lawrence Berkeley National Laboratory

University of California, Berkeley, CA 94720

June 1995

This work was supported by the Director, Office of Energy Research, Division of Nuclear Physics of the Office of High Energy and Nuclear Physics of the U.S. Department of Energy under Contract DE-AC03-76SF00098

Detrouton of THS Documert 
E) recycios paper 


\section{DISCLAIMER}

This report was prepared as an account of work sponsored by an agency of the United States Government. Neither the United States Government nor any agency thereof, nor any of their employees, make any warranty, express or implied, or assumes any legal liability or responsibility for the accuracy, completeness, or usefulness of any information, apparatus, product, or process disclosed, or represents that its use would not infringe privately owned rights. Reference herein to any specific commercial product, process, or service by trade name, trademark, manufacturer, or otherwise does not necessarily constitute or imply its endorsement, recommendation, or favoring by the United States Government or any agency thereof. The views and opinions of authors expressed herein do not necessarily state or reflect those of the United States Government or any agency thereof. 


\section{DISCLAIMER}

Portions of this document may be illegible in electronic image products. Images are produced from the best available original document. 


\title{
P-TYPE SILICON DRIFT DETECTORS
}

\author{
J.T. Walton, B. Krieger, D. Krofcheck, R. O'Donnell, \\ G. Odyniec, M.D. Partlan, N.W. Wang \\ Lawrence Berkeley National Laboratory, University of California, \\ Berkeley, CA, 94720, USA
}

\begin{abstract}
Preliminary results on $16 \mathrm{~cm}^{2}$, position-sensitive silicon drift detectors, fabricated for the first time on p-type silicon substrates, are presented. The detectors were designed, fabricated, and tested recently at LBL and show interesting properties which make them attractive for use in future physics experiments.

A pulse count rate of approximately $8 \times 10^{6} \mathrm{~s}^{-1}$ is demonstrated by the p-type silicon drift detectors. This count rate estimate is derived by measuring simultaneous tracks produced by a laser and photolithographic mask collimator that generates double tracks separated by $50 \mu \mathrm{m}$ to $1200 \mu \mathrm{m}$.

A new method of using ion-implanted polysilicon to produce precise valued bias resistors on the silicon drift detectors is also discussed.

This work was supported by the Director, Office of Energy Research, Division of Nuclear Physics of the Office of High Energy and Nuclear Physics of the U.S. Department of Energy under Contract DE-AC03-76SF0098.
\end{abstract}




\section{INTRODUCTION}

In recent years, position-sensitive silicon detectors have become indispensable in high energy physics experiments. However, the use of silicon strip detectors for tracking in high multiplicity environments is complicated by so-called ghost hits (ambiguities of combined one-dimensional information collected from multiple planes of strip detectors), and the use of silicon pixel detectors is expensive due to their large number of readout channels. An ideal detector for the high multiplicity collisions appears to be the silicon drift detector (SiDD) $[1,2]$. Notable features of the silicon drift detector, besides its high position and energy resolution and very good two track resolution, include its small $(\sim 0.1 \mathrm{pF})$, area-independent output capacitance, modest readout electronics requirements, and minimal need for cooling.

In this paper, we describe our work on SiDDs which have been fabricated on p-type silicon rather than on the more typically used n-type silicon. We have investigated the use of p-type silicon as the starting material for SiDDs because of several benefits possibly to be gained. High purity, p-type silicon grown by the float-zone process exhibits better radial dopant uniformity than n-type, float-zone silicon. High purity, n-type silicon with the dopant uniformity required for position-sensitive SiDDs must be initially grown as very pure p-type material via the float-zone technique and subsequently neutron transmutation doped. Neutron transmutation doped silicon is expensive and available only by special order; float-zone, p-type silicon, on the other hand, is readily available and relatively inexpensive. In addition, float-zone silicon is free of the radiation damage caused by the neutron transmutation doping process; the response of neutron-induced defects to thermal annealing is complicated and can result in uncontrollable changes in material resistivity during detector processing [3]. Moreover, there has been some evidence recently that ptype silicon is more radiation-resistant than n-type [4]. In the following sections, we describe the structure of the p-type silicon drift detector (pSiDD), the characterization of its position resolution, and the development of ion-implanted polycrystalline silicon resistors for biasing the detector.

\section{DEVICE DESCRIPTION}

We fabricated the pSiDDs on 3 " diameter, $300 \mu \mathrm{m}$ thick, (111), $7 \mathrm{k} \Omega$-cm, p-type, floatzone silicon. The process used to fabricate the detectors is described in detail elsewhere [5]. Figure 1 shows the structure of the position-sensitive $\mathrm{pSiDD}$ developed at LBL; the detector geometry was adapted from an n-type SiDD (nSiDD) designed by P. Rehak at 
Brookhaven National Laboratory. In this work, the nomenclature of the n-type SiDDs has been retained, and thus we will refer to the $\mathrm{n}^{+}$contacts as "cathodes" and the $\mathrm{p}^{+}$contacts as "anodes." The pSiDD consists of 332 parallel cathodes formed on both sides of a p-type silicon substrate. The cathode pitch is $120 \mu \mathrm{m}$. At one end of the detector is a row of 178 anodes with $250 \mu \mathrm{m}$ pitch. Around the active region is a high voltage guard region consisting of $30 \mu \mathrm{m}$ wide $\mathrm{n}^{+}$strips. The guard structure is tapered so that there are more guard strips protecting the cathodes farther from the anodes (which will need to withstand higher voltages) than the cathodes nearer the anodes.

During detector operation, the cathodes are reverse biased to deplete the entire silicon substrate and to create a uniform electric field in the direction parallel to the surface. When electron-hole pairs are created by ionizing radiation passing through the detector, the electrons are quickly collected at the reverse biased cathodes. The holes, on the other hand, are focused down the center plane of the detector and drift at constant velocity toward the row of anodes at the low voltage end of the device. The transit time of the holes allows the distance of the incident radiation from the anode plane to be calculated. The distribution of the signal charge over the readout anodes gives the second position coordinate.

\section{DETECTOR CHARACTERIZATION}

We have studied the performance of the pSiDDs using measurements of a) the drift time vs. position of the incident radiation (i.e., the linearity of the detector response), b) energy resolution, and c) single-track and double-track position resolution. In the following sections, we discuss the results of each type of measurement.

At this point, we would like to stress that the nSiDD and pSiDD should have identical position resolution capabilities. This comment is based on the assumption that the spatial growth of the signal charge packet (either electrons or holes) as it drifts down the detector is governed by diffusion. Based on this assumption, it is readily shown that, at room temperature, the rms spatial spread in the packet width, $\sigma(x)$, is given by

$$
\begin{aligned}
\sigma(\mathrm{x}) & =\sqrt{2 \mathrm{Dt}} \\
& =\sqrt{\frac{2 \mathrm{kT} \mu}{\mathrm{q}} \frac{\mathrm{X}_{0}}{\mu \mathrm{E}}} \\
\sigma(\mathrm{x}) & =0.228 \sqrt{\frac{\mathrm{X}_{0}}{\mathrm{E}}} \stackrel{(\mathrm{cm})}{ }
\end{aligned}
$$


where $X_{0}$ is the distance (in $\mathrm{cm}$ ) of the incident particle's point of interaction on the drift detector from the collecting anode, and $\mathrm{E}$ is the applied electric drift field (in $\mathrm{V} / \mathrm{cm}$ ), and is independent of any material properties. However, the speed with which the packet arrives at the collecting anodes is dependent on the carrier mobility, which is approximately three times less for holes than electrons. Therefore, if the drift detector signals were digitized at the anodes, the digitizing rate for the pSiDD could be about $\left(\mu_{\mathrm{h}} / \mu_{\mathrm{e}}\right)$ times slower than for a nSiDD for the same number of sample points. Note also that the pSiDD peak amplitude would be $\left(\mu_{\mathrm{h}} / \mu_{\mathrm{e}}\right)$ smaller than the nSiDD peak.

\section{a) Drift time vs. Position}

We have demonstrated that the pSiDDs collect signal charge across their entire $4 \mathrm{~cm}$ length, and that the drift time is a linear function of the incident position of the excitation [5]. Accurate measurements of the transit time as a function of the distance that the charge cloud drifts were achieved by attaching the detectors to a computer-controlled $x-y$ stage that could be stepped in $0.5 \mu \mathrm{m}$ increments. Infrared light $(\lambda=1.06 \mu \mathrm{m})$ from a pulsed Nd:YAG laser was focused through a microscope objective to a $5 \mu \mathrm{m}$ diameter spot on the detector. Figure 2 shows the relation between the signal arrival time and laser position over a $7 \mathrm{~mm}$ distance, with an applied drift field of $263 \mathrm{~V} / \mathrm{cm}$. The relation is very linear, and its slope agrees with the expected drift velocity at this electric field to within $2 \%$.

\section{b) Energy Resolution}

For energy resolution measurements, we placed an ${ }^{241} \mathrm{Am}$ source directly over the anode region and fed the charge signal through a hybrid preamplifier and a variable time constant amplifier to a multichannel analyzer. With a $0.25 \mu \mathrm{sec}$ shaping amplifier time constant, we obtained an energy resolution of $3.04 \mathrm{keV} \mathrm{FWHM}$ at $59.4 \mathrm{keV}$. The ${ }^{241} \mathrm{Am}$ spectrum is shown in Figure 3. The noise contribution of the electronics alone, measured using a pulse generator input at the preamplifier, was $2.95 \mathrm{keV}$ FWHM. This suggests that the pSiDDs can provide excellent energy measurement when equipped with lower noise electronics.

\section{c) Single-Track and Double-Track Position Resolution}

In our single-track and double-track measurements of the $\mathrm{PSiDD}$ performance we used the electronics described above. For determination of the system time resolution, which we used to assess the position resolution in the drift dimension, we added a time-to-amplitude converter (TAC) module. The TAC, when connected to the multichannel analyzer, allowed us to make the required system time resolution measurements. 


\section{i) Single-track resolution}

To be able to determine the position of a 'hit' on a SiDD, two 'times' are needed - the 'time-zero' at which the particles hit the $\mathrm{SiDD}$, $t_{0}$, which is typically provided by another detector in the system, and the time that the hit signal arrives at the SiDD anodes, $\mathrm{t}_{\mathrm{s}}$. The position, $\mathrm{X}_{\mathrm{o}}$, of the hit on the SiDD is then calculated from:

$$
\begin{aligned}
X_{o} & =\left(t_{s}-t_{0}\right) v_{\text {drift }} \\
& =\left(t_{s}-t_{0}\right) \mu E,
\end{aligned}
$$

where $\mu$ is the carrier mobility and $\mathrm{E}$ is the internal electric drift field. In the following we assume that any error in determining the position, $X_{o}$, is directly due to errors in determining $t_{s}$, or

$$
\Delta \mathrm{X}_{\mathrm{o}} \sim \Delta \mathrm{t}_{\mathrm{s}} .
$$

The rms system time resolution, $\Delta \mathrm{t}_{\mathrm{S}}$, is dependent on the signal level, $\mathrm{V}_{\boldsymbol{S}}$, the rms system noise, $V_{\mathrm{n}}$, and shaping amplifier time constant, $\tau$, as follows:

$$
\Delta \mathrm{t}_{\mathrm{s}} \approx\left(\mathrm{V}_{\mathrm{n}} / \mathrm{N}_{\mathrm{s}}\right) 2 \tau
$$

providing $V_{n} \ll V_{S}$, and $t_{r} \ll \tau$, where $t_{r}$ is the input signal rise time. Since, at short shaping amplifier time constants,

$$
\mathrm{V}_{\mathrm{n}} \sim \frac{1}{\sqrt{\tau}}
$$

then

$$
\Delta \mathrm{t}_{\mathrm{s}} \sim \sqrt{\tau}
$$

Therefore, the single-track time resolution should improve slowly with decreasing amplifier time constants. As will be discussed, however, the SiDD anode signal itself presents a lower limit on $\tau$.

We have summarized our system measurements in Table $\mathrm{I}$ as well as including an estimate of the limiting single-track position resolution based solely on system electronics performance. A few comments about Table I are required: 
1. The system resolution measurements were obtained from the width of the $59.4 \mathrm{keV}$ ${ }^{241}$ Am peak.

2. The $\Delta t_{S}$ calculated is obtained from Eq. 4 above and assumes a signal of $60 \mathrm{keV}$.

3. The position resolution is calculated for a $\mathrm{PSiDD}$ using the product of the hole mobility $\left(480 \mathrm{~cm}^{2} / \mathrm{V}-\mathrm{sec}\right)$, the electric field $(500 \mathrm{~V} / \mathrm{cm}$, the magnitude of the field used in these measurements) and the calculated time resolution.

Figure 4 shows a plot of the expected rms time variance, $\sigma(t)$, as a function of the detector length. The upper curve in Figure 4 is the calculated rms spatial broadening as a function of drift distance from the anodes; note that for a given distance the spatial broadening is identical for both electrons and holes. The lower two curves represent the calculated rms time spread as a function of drift distance from the anodes for n-type and ptype SiDDs; note that the time spread is different for electrons and holes by a factor of $\left(\mu_{\mathrm{h}} / \mu_{\mathrm{e}}\right)$.

The spatial resolutions in Table I were calculated by neglecting that the signals from the SiDD anodes are Gaussian functions of time [6] and have a finite rise time. The anode signal rise time depends on the time variance as follows:

$$
t_{r} \approx \sigma(t)
$$

The significant change in $\sigma(\mathrm{t})$ across the $4 \mathrm{~cm}$ long pSiDD does not permit, for optimum timing, the use of a fixed amplifier time constant. To obtain the optimum timing the amplifier time constant should be matched to the signal rise time, or

$$
2 \tau \approx \sigma(t)
$$

Comparison of the calculated spatial resolution in Table I with the expected spatial variance in Figure 4 shows that our electronics, when approximately matched to the expected anode signal rise time, does not contribute significantly to our measurements. We make use of this result in our estimates of double-track resolution in the following section.

\section{ii) Double-track resolution measurements}

To examine two-track resolution which is not affected by the position dependence of time variance, we have chosen to measure it over a limited distance near the collecting anodes. The results presented here are included to show the potential of the pSiDDs and 
the technique we have used, and not to provide a definitive answer on the double-track resolution capabilities of the pSiDDs.

In making the double-track resolution measurements one needs to keep in mind that the charge sensitive preamplifier used imposes certain limitations, namely that it integrates the Gaussian signal from the pSiDD anodes. Figures $5 \mathrm{a}$ \&b show two simulated Gaussian pulses separated by $3 \sigma$ and $4 \sigma$, respectively. The dashed line represents the integral of the Gaussians. This demonstrates that a separation of more than $3 \sigma$ is needed for the charge sensitive preamplifier to resolve two peaks without further signal processing techniques.

Our experimental arrangement for making the double track measurements is shown in the insert of Figure 6. The glass plate in the figure is a chrome photolithography mask on which a pattern of $5 \mu \mathrm{m} \times 100 \mu \mathrm{m}$ holes was etched. The photomask is aligned to the detector such that the $100 \mu \mathrm{m}$ hole dimension is parallel to the pSiDD cathode stripes; the photomask can be placed directly on the detector, pattern side down, since the mask is made of insulating chrome oxide. The laser is defocused to spread light over several of the pinholes in the opaque chrome, in order to simulate multiple simultaneous tracks through the pSiDD. The intensity of the laser was adjusted to generate charge signals approximately equivalent to those deposited by minimum ionizing particles.

Results of this measurement are shown in Figure 6. The shaping amplifier time constant used in these measurements was $20 \mathrm{~ns}$. Four distinct peaks can be seen, corresponding to signals coming through seven pinholes in the mask. The holes are spaced at intervals of $50,100,150,300,600$ and $1200 \mu \mathrm{m}$. The peaks centered at $336 \mathrm{~ns}, 568 \mathrm{~ns}$, and $1053 \mathrm{~ns}$ are due to light entering through single holes. The feature centered at $148 \mathrm{~ns}$ represents a superposition of signals from the four holes spaced 50,100, and $150 \mu \mathrm{m}$ away from each other. Thus, the smallest separation between two pinholes which could be resolved was $300 \mu \mathrm{m}$. The placement of the chrome mask on the detector (at about $4 \mathrm{~mm}$ from the anodes) was such that the time spread, $\sigma(t)$, was $\sim 40$ ns.

As seen in Figure 5, the holes in the plate must be more than $3 \sigma(t)(120 \mathrm{~ns}$ or $300 \mu \mathrm{m})$ apart in order to be resolved by these electronics. Therefore, the results at small pinhole spacings in Figure 6 are consistent the discussion above.

The solid line in Figure 6 shows a fit of four Gaussians to the data. The width of the Gaussians is $65 \mathrm{~ns}$. As shown in Table II, the fitted centroids of the signals match the time separations calculated from the known spacings between the pinholes very well. This result confirms our estimates in Table I that our electronics system noise would not influence the double-track measurements. 


\section{DEVELOPMENT OF POLY-SI VOLTAGE DIVIDER}

We have developed a process for fabricating a polycrystalline silicon (poly-Si) voltage divider on the pSiDDs. Since the position resolution of the SiDD depends on maintaining a constant carrier drift velocity and thus a constant drift field, the voltage divider resistors must be uniform. There have been several approaches to integrating bias resistors on silicon drift detectors. The Brookhaven n-type SiDD design used lightly ion implanted regions at the edges of the cathodes to make a resistor chain on the detector [7]. Other resistor integration schemes $[8,9]$ have utilized the repeating MOSFET-like structure of the cathodes by depositing a metal gate over the $\mathrm{SiO}_{2}$ region separating the cathodes.

The poly-Si approach has several advantages over the techniques mentioned above: e.g., poly-Si can better withstand high voltages than lightly implanted silicon resistors which are likely to exhibit punchthrough, and poly-Si resistors are neither voltage-dependent nor constrained within a small range of operating voltages, as they are with the MOS voltage dividers. Furthermore, it was believed that poly-Si would yield better uniformity of resistances than the other techniques, due to the good thickness control attainable with the low pressure chemical vapor deposition method used. Poly-Si resistors have been used for integrated circuits $[10,11]$ and silicon strip detectors.

The resistivity of poly-Si can be varied between $\sim 10^{6}$ and $\sim 10^{-3} \Omega-\mathrm{cm}$ by varying dopant concentration [12], and thus easily allows fabrication of resistors within our target range of $200-300 \mathrm{k} \Omega$. This range of resistance values was determined by compromising between the need for a high resistance value to limit power dissipation and the need for a lower resistance value so that small fluctuations in resistor current do not lead to large voltage fluctuations. A main concern in designing the fabrication process to yield precisevalued resistors was that the resistivity of poly-Si varies very rapidly with dopant concentration, so that small fluctuations in dopant concentration would result in large variations in resistor values. For this reason, ion implantation was chosen as the doping method, since it allows good control over dopant concentration and uniformity.

The proposed structure of the poly-Si resistor network, as it will be incorporated on to the $\mathrm{pSiDD}$, is shown in Figure 7. Low temperature chemical vapor deposition is used to deposit a $\mathrm{SiO}_{2}$ spacer layer over the detector after the $\mathrm{n}^{+}$and $\mathrm{p}^{+}$regions have been patterned and to deposit an undoped amorphous $\mathrm{Si}$ layer over the $\mathrm{SiO}_{2}$. The amorphous $\mathrm{Si}$ layer is ion implanted with phosphorus and then annealed to activate the dopants and to yield polycrystalline microstructure. The resistors are then patterned and etched. Contacts between the resistors and cathodes are formed by etching windows in the low temperature 
$\mathrm{SiO}_{2}$ layer and depositing and patterning $\mathrm{Al}$ contacts. Resistors fabricated using this process have been tested alone but not as yet integrated on to the detectors.

To determine the phosphorus implant dose, we fabricated resistors using four different implant levels. The sheet resistance vs. implant dose is shown in Figure 8. The data indicate that the sheet resistance varies less rapidly at higher doses, which means that, for higher doses, local fluctuations in implant dose will have less effect on resistance. The resistors fabricated using this technique were quite uniform; the measured variation in sheet resistance over an $8 \mathrm{~cm}^{2}$ area was $\pm 2 \%$ at $1 \times 10^{14} \mathrm{~cm}^{-2}$ and $\pm 1 \%$ at $1 \times 10^{15} \mathrm{~cm}^{-2}$.

\section{CONCLUSIONS}

We have highlighted some of our recent research and development on p-type silicon drift detectors. In particular, we have demonstrated a novel technique for measuring the doubletrack resolution capabilities of these detectors and have also shown results on a possible technique for fabricating the precise, high-valued resistors needed for biasing the detectors.

Using "off-the-shelf" electronics we have been able to resolve double tracks separated by about $300 \mu \mathrm{m}$ on the detector, which corresponds to a count rate of about $8 \mathrm{MHz}$. As noted in the paper, this rate was limited by our use of a charge sensitive preamplifier, not by the p-type silicon drift detector itself.

The excellent resistor uniformity from the ion-implanted polysilicon process described should allow creation of more uniform drift electric fields than we have presently been able to realize. Using the double-track measurement capability that we have developed, we expect shortly to confirm the viability of the polysilicon resistor approach.

\section{ACKNOWLEDGMENTS}

We thank P. Rehak of Brookhaven National Laboratory for allowing us access to his detector mask designs, and S.E. Holland of Lawrence Berkeley Laboratory for his advice on polysilicon resistor fabrication. 


\section{FIGURE CAPTIONS}

Figure 1. Schematics showing a) cross-sectional view, and b) top view of the pSiDD.

Figure 2. Drift time versus position of the incident laser pulse.

Figure 3. ${ }^{241} \mathrm{Am}$ spectrum measured with the pSiDD. The energy deposited by the $59.4 \mathrm{keV}$ gamma-ray is about $2 / 3$ that of a minimum ionizing particle passing through $300 \mu \mathrm{m}$ of silicon.

Figure 4. The calculated rms spatial broadening (top curve) and the calculated rms time spread (lower two curves) as a function of drift distance from the anodes. The spatial broadening is identical for both n-type and p-type SiDDS, whereas the spread in time is different by a factor of $\left(\mu_{\mathrm{h}} / \mu_{\mathrm{e}}\right)$. An electric field of $500 \mathrm{~V} / \mathrm{cm}$ was assumed for the calculations. This figure is similar to that of Fig. 10 in Ref. [6].

Figure 5. Simulated Gaussian pulses separated by $3 \sigma$ and $4 \sigma$. The dashed line represents the integral of the Gaussians. This illustrates that a separation of more than $3 \sigma$ is needed for the charge sensitive preamplifier to resolve two peaks without further signal processing techniques.

Figure 6. Signals measured by using a $\mathrm{Cr}$ photomask to collimate infrared light for simulating multiple tracks in the $\mathrm{pSiDD}$. The insert shows a schematic of the technique. The laser is defocused to illuminate an array of $5 \mu \mathrm{m} \times 100 \mu \mathrm{m}$ holes spaced at intervals of $50,100,150,300,600$ and $1200 \mu \mathrm{m}$. The measured peaks centered at $336 \mathrm{~ns}, 568 \mathrm{~ns}$, and $1053 \mathrm{~ns}$ are due to light entering through single holes. The feature centered at $148 \mathrm{~ns}$ represents a superposition of signals from four holes. The amplifier shaping time was $20 \mathrm{~ns}$.

Figure 7. Schematics of a) top view and b) cross section of the poly-Si resistors as they will be integrated on the pSiDDs.

Figure 8. Measurements of sheet resistance vs. phosphorus implant dose. The variation in sheet resistance over an $8 \mathrm{~cm}^{2}$ area was $\pm 2 \%$ at $1 \times 10^{14} \mathrm{~cm}^{-2}$ and $\pm 1 \%$ at $1 \times 10^{15} \mathrm{~cm}^{-2}$. 
Table I

Single-Track Timing and Position Resolution

\begin{tabular}{|c|c|l|l|c|}
\hline $\begin{array}{c}\text { Amplifier } \\
\text { Time Constant }\end{array}$ & $\begin{array}{c}\text { System } \\
\text { FWHM } \\
\text { measured }\end{array}$ & $\begin{array}{l}\Delta \mathrm{t}_{\mathrm{s}} \\
\mathrm{rms} \\
\text { calculated }\end{array}$ & $\begin{array}{l}\Delta \mathrm{t}_{\mathrm{s}} \\
\text { rms } \\
\text { measured }\end{array}$ & $\begin{array}{l}\text { Position } \\
\text { resolution, rms, } \\
\text { calculated }\end{array}$ \\
\hline $100 \mathrm{~ns}$ & $4.3 \mathrm{keV}$ & $4.2 \mathrm{~ns}$ & $2.0 \mathrm{~ns}$ & $10.5 \mu \mathrm{m}$ \\
\hline $50 \mathrm{~ns}$ & $6.0 \mathrm{keV}$ & $3.2 \mathrm{~ns}$ & $1.6 \mathrm{~ns}$ & $8.0 \mu \mathrm{m}$ \\
\hline $20 \mathrm{~ns}$ & $9.6 \mathrm{keV}$ & $1.9 \mathrm{~ns}$ & $1.2 \mathrm{~ns}$ & $4.7 \mu \mathrm{m}$ \\
\hline
\end{tabular}

Table II

Comparison of Times Between Signals (from Gaussian Fit) and Time Calculated from Pinhole Spacing

\begin{tabular}{|c|l|l|l|}
\hline Pinhole spacing & $475 \mu \mathrm{m}^{*}$ & $600 \mu \mathrm{m}$ & $1200 \mu \mathrm{m}$ \\
\hline $\begin{array}{c}\text { Calculated time } \\
\text { separation }\end{array}$ & $190 \mathrm{~ns}^{*}$ & $240 \mathrm{~ns}$ & $480 \mathrm{~ns}$ \\
\hline $\begin{array}{c}\text { Measured time } \\
\text { separation }\end{array}$ & $188 \mathrm{~ns}$ & $232 \mathrm{~ns}$ & $485 \mathrm{~ns}$ \\
\hline
\end{tabular}

*Note: The spacing was averaged over the pinholes that appear in this peak. Based on this average spacing the time was calculated using a hole mobility of $500 \mathrm{~cm}^{2} / \mathrm{V}-\mathrm{s}$ and and electric field of $500 \mathrm{~V} / \mathrm{cm}$. 
[1] E. Gatti and P. Rehak, Nucl. Instr. and Meth. 225 (1984) 608.

[2] E. Gatti, P. Rehak, and J.T. Walton, Nucl. Instr. and Meth. 226 (1984) 129.

[3] J.C. Corelli and J.W. Corbett, "Impact of Defects Formed in Neutron Transmutation Doping of Silicon on Device Performance," in Neutron Transmutation Doped Silicon, ed. J. Guldberg (Plenum Press, 1981) p. 35.

[4] E. Beuville et al., Nucl. Instr. and Meth. A288 (1990) 68.

[5] N.W. Wang et al., IEEE Trans. Nucl. Sci., to be published.

[6] E. Gatti, A. Longoni, P. Rehak, M. Sampietro, Nucl. Instr. and Meth. A253 (1987) 393.

[7] W. Chen, H.W. Kraner, Z. Li and P. Rehak, IEEE Trans. Nucl. Sci. 41 (1994) 941.

[8] A. Bischoff et al., Nucl. Instr. and Meth. A326 (1993) 27.

[9] P. Lechner et al., Proc. of the 6th European Symposium on Semiconductor Detectors, Milan, February 1992, MPI-PhE/92-12.

[10] N. Lu, L. Gerzberg, C. Y. Lu., and J. D. Meindl, IEEE Trans. Elec. Dev. ED-28 (1981) 812 .

[11] T. Ohzone et al., IEEE Trans. Elec. Dev. ED-32 (1985) 1749.

[12] T.I. Kamins, Polycrystalline Silicon for Integrated Circuit Applications (Kluwer Academic Press, 1988). 


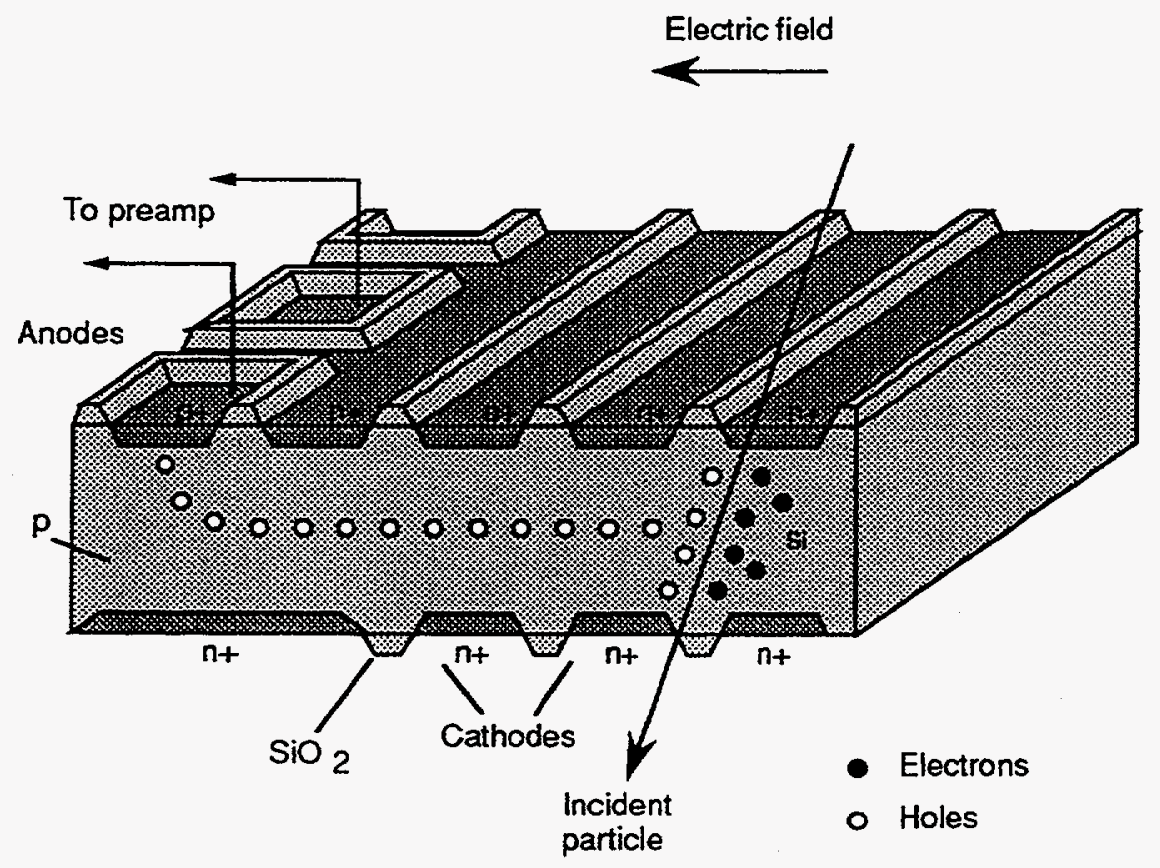

Figure $1 \mathrm{a}$. 


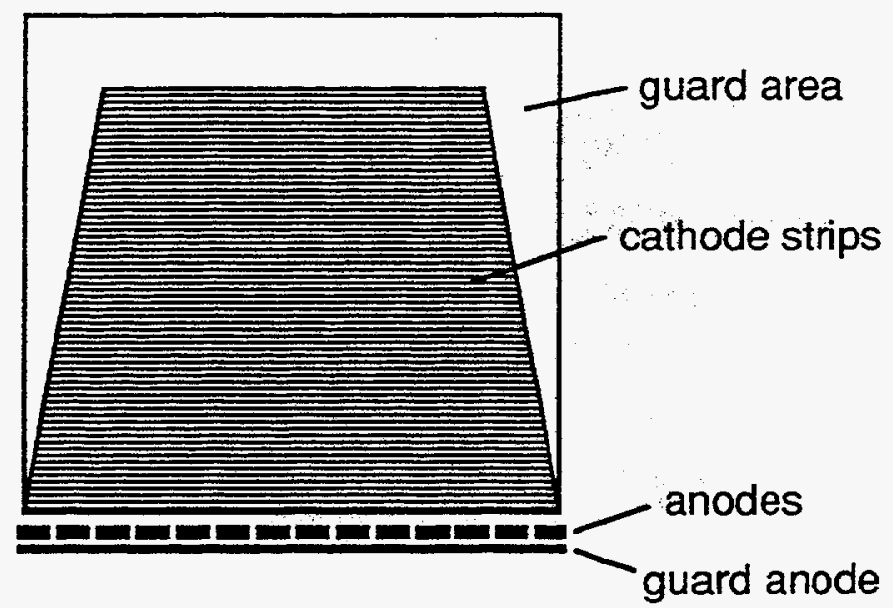

Figure $1 b$. 


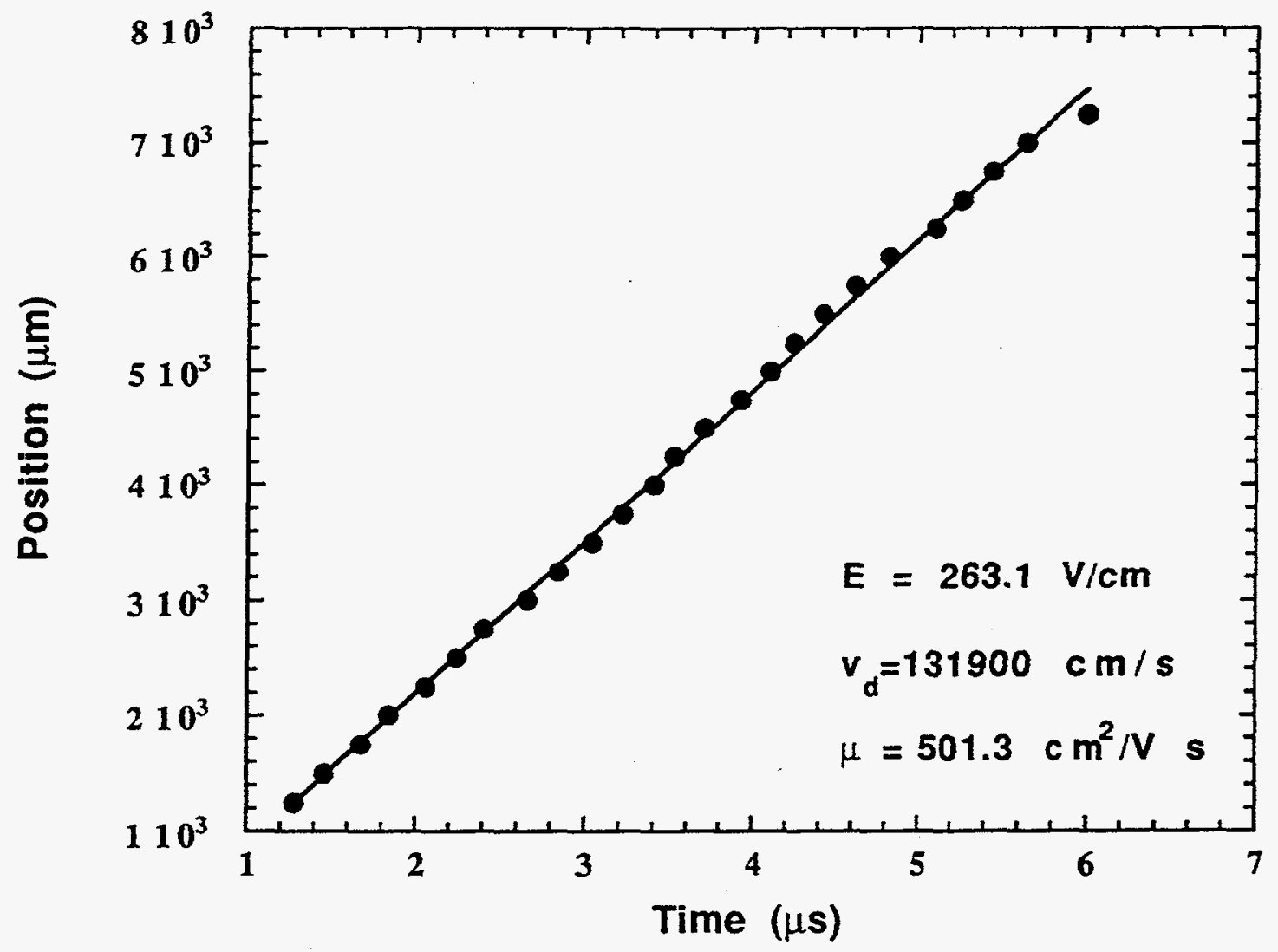

Figure 2 


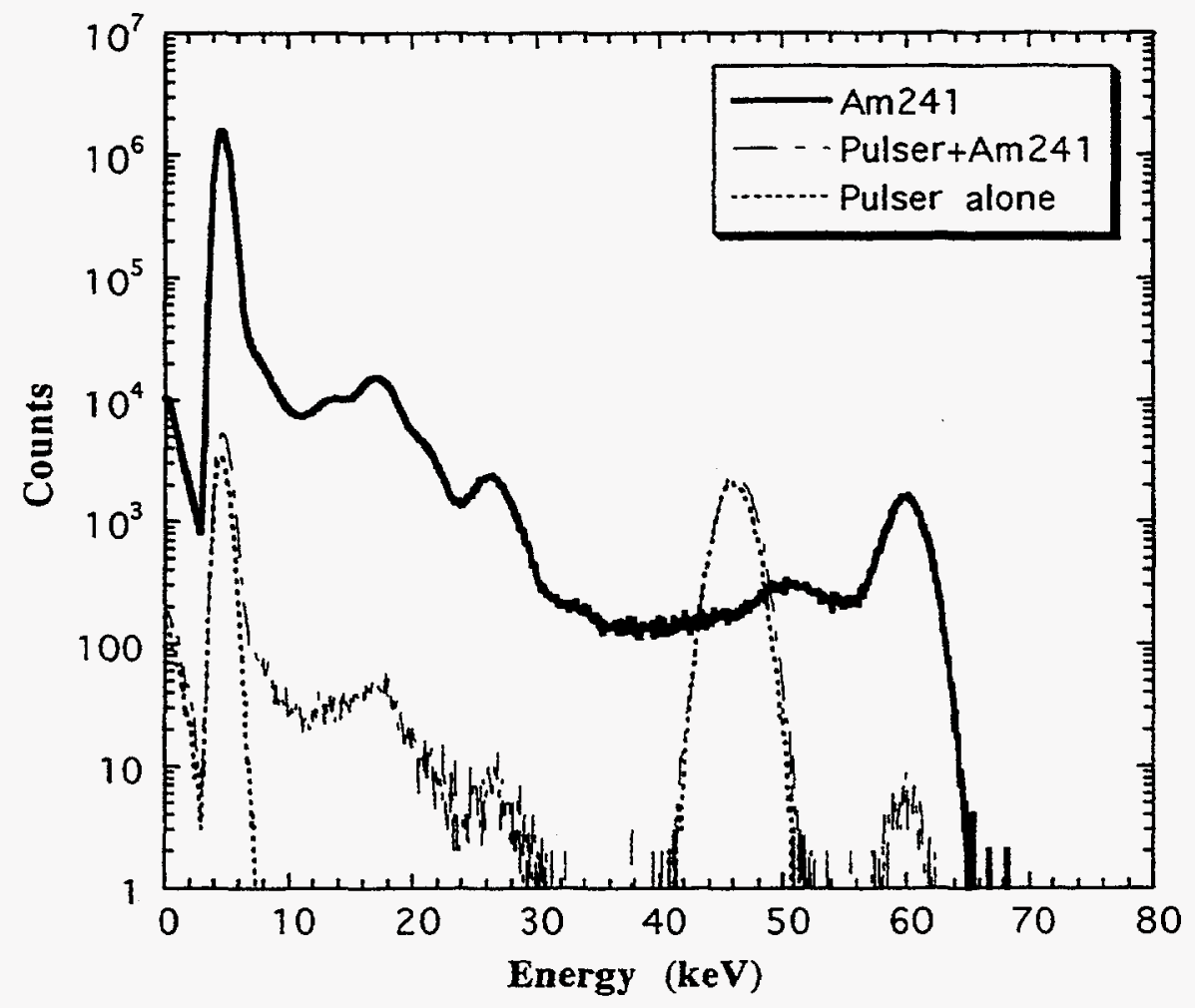

Figure 3 


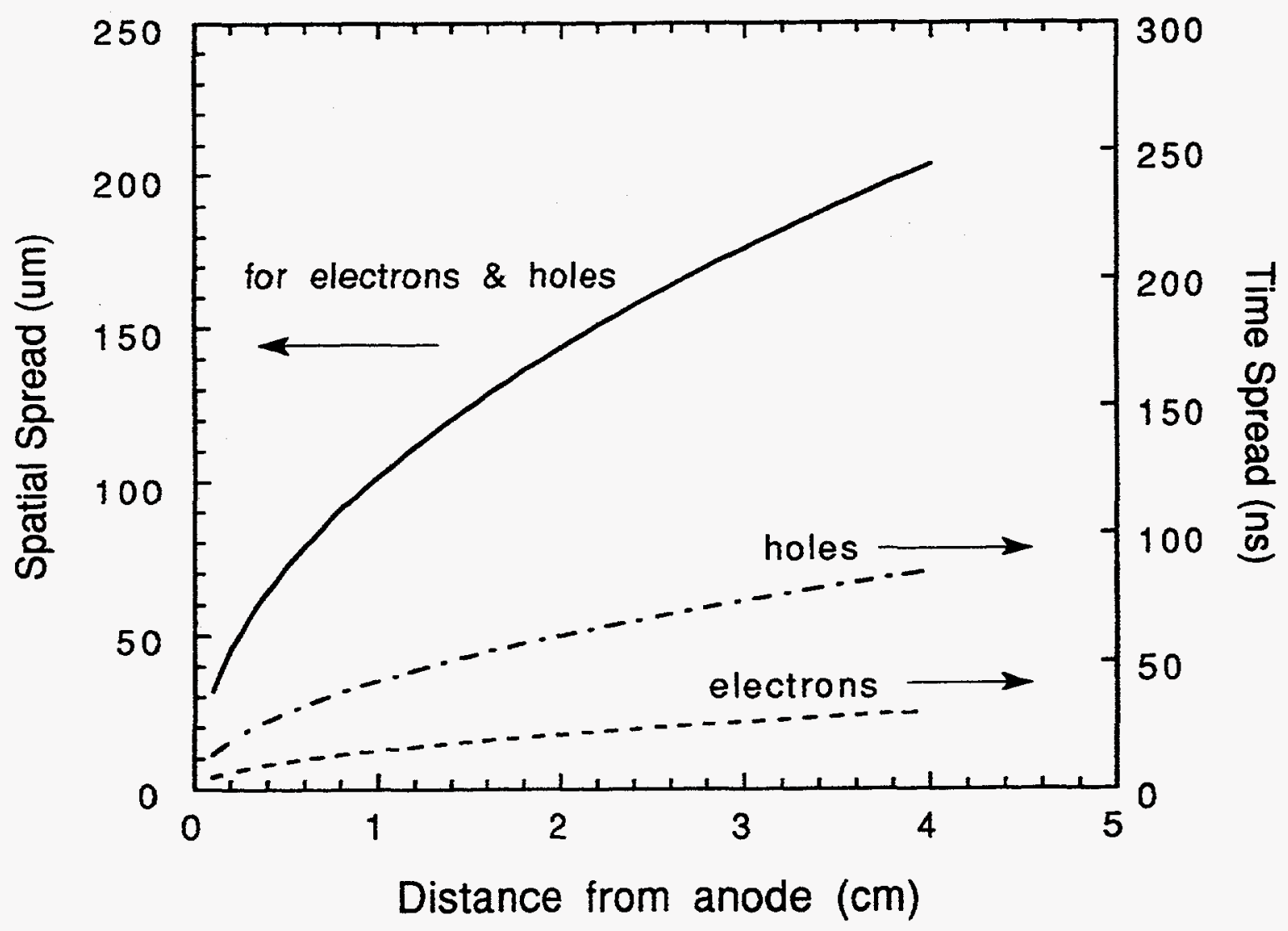

Figure 4 

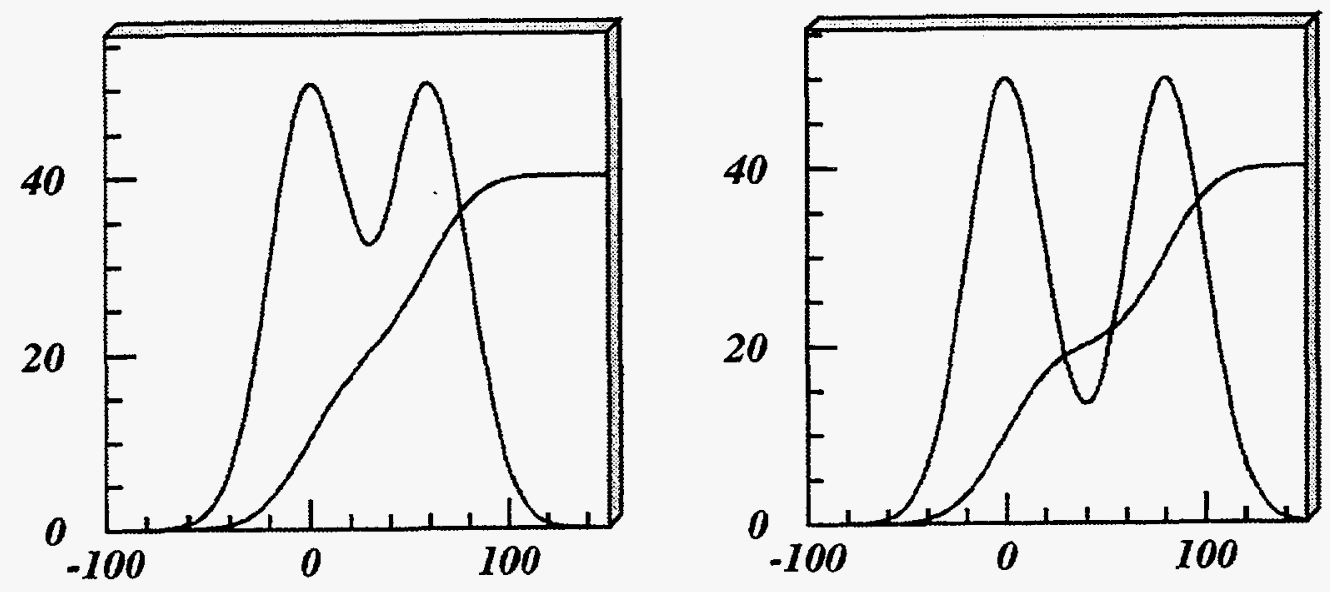

Figure $5 a$.

Figure 5b. 


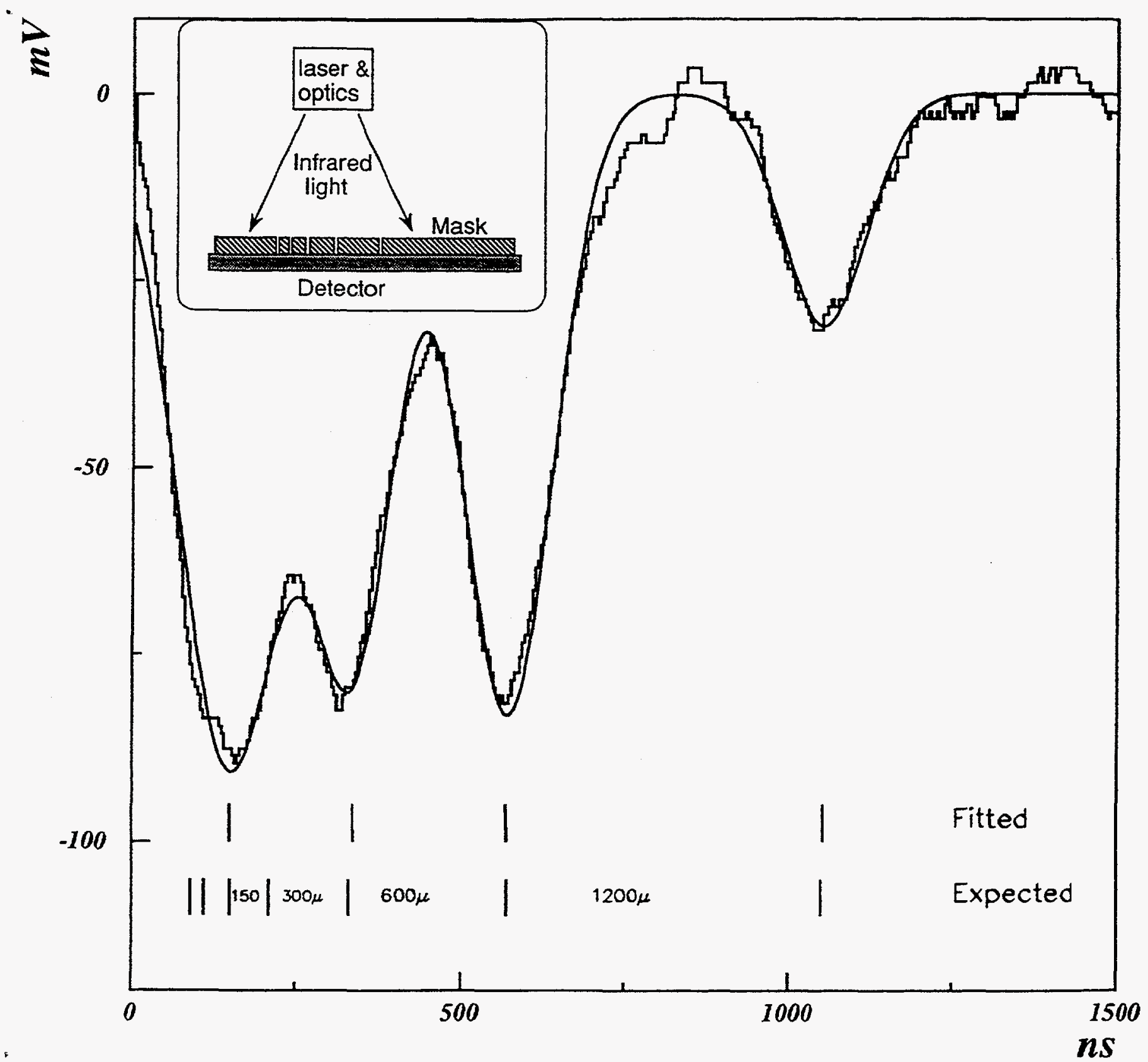

Figure 6 


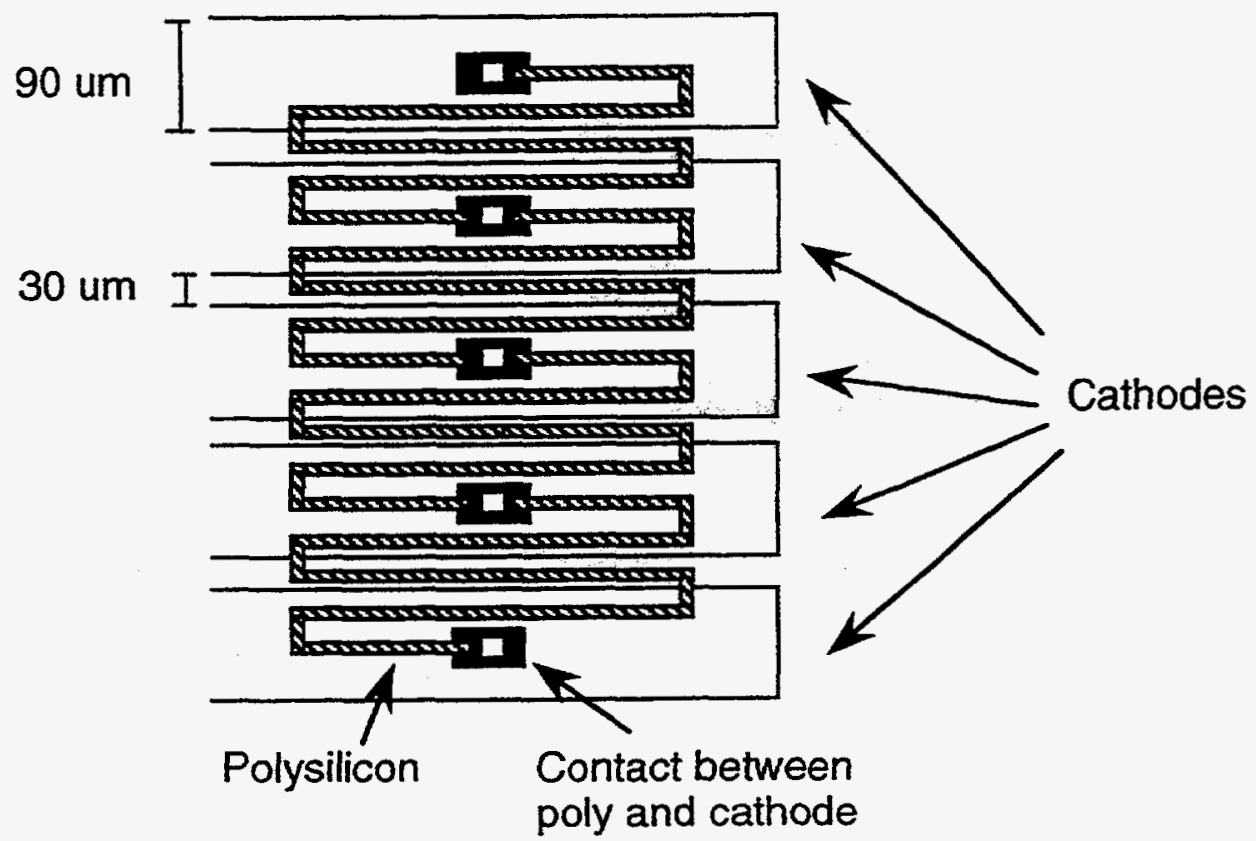

Figure $7 a$. 


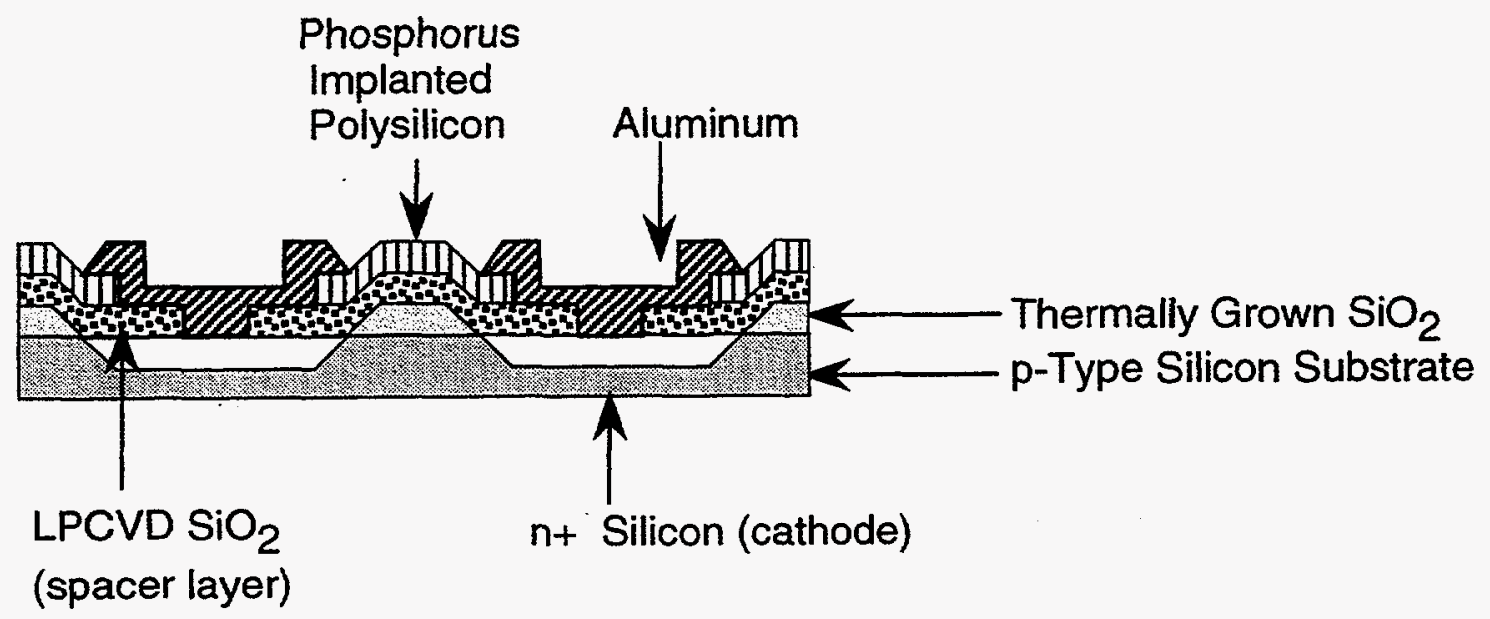

Figure $7 b$. 


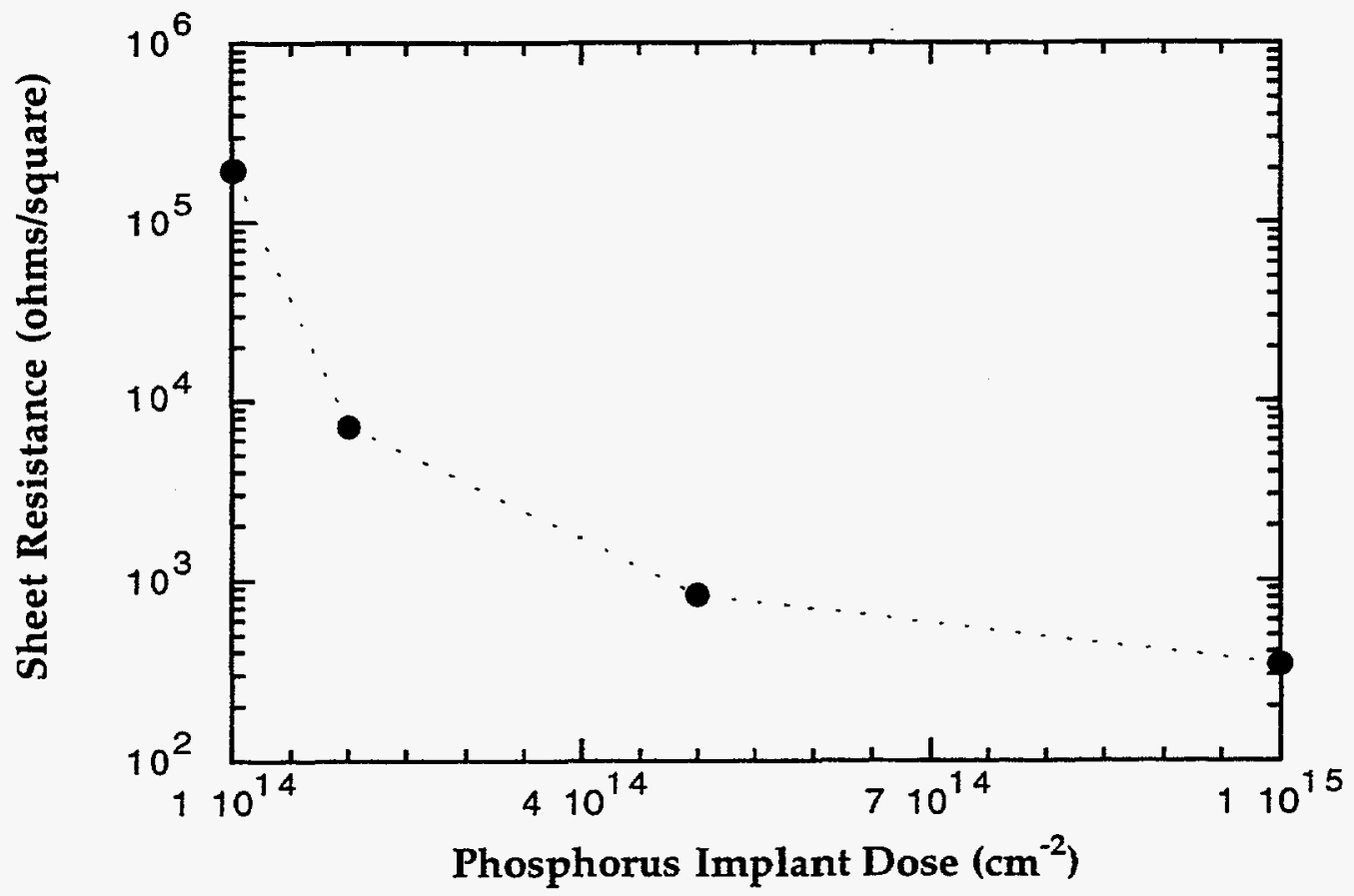

Figure 8 